\section{Perforations after routine biopsy in IBD patients, their management and potential risk reductions by microscopic imaging with endocytoscopy}

\section{To the Editor,}

Biopsy-related perforation is a rarely reported complication of colonoscopy and controversy remains whether diagnostic biopsy confers also an increased risk for perforation in patients with inflammatory bowel disease (IBD) [1-3]. In this letter, as exemplified by a case report, we aim to raise awareness for biopsy-related perforations in longstanding ulcerative colitis (UC) even in patients without overt inflammation and at the same time provide an outlook on the potential of endoscopic techniques enabling real-time in vivo microscopy such as endocytoscopy (EC).

Our patient was a 77-year-old male with steroid dependant pancolitis since 2002 and an increase in clinical activity; overall the Mayo Clinical Score (MCS) was 6. While high definition colonoscopy demonstrated a Mayo Endoscopic Score (MES) of 1 , in vivo microscopic imaging using a latest generation endocytoscope (Olympus CF-H290ECI, Olympus, Japan) showed crypt distension and deformity along with inflammatory cell infiltrates and hypervascularisation (Fig. 1A). After biopsy in the sigmoid colon with a standard forceps, a small perforation was immediately visualized at the site of physical biopsy (Fig. 1B). An over-the-scope clip (OTSC, Ovesco, Germany) was mounted onto the endoscope and the perforation was successfully closed by over-the-scope clipping (Fig. 1C). Conventional histopathology showed fibrosis of the lamina propria along with atrophy of the entire colonic wall and inflammatory cell infiltrates (Fig. 1D). Six months after colonoscopy, the patient was in stable clinical and steroid-free remission under infliximab.

Perforation rates during diagnostic colonoscopy in IBD patients show considerable variation across reports. Contrastingly to 3 perforations in a large prospective study with 558 patients, which were most likely associated with persistent disease or complicated disease behaviour, no increased incidence of perforation in IBD patients had been reported [4]. Analysing over 33,000 IBD related hospitalizations, it was found that IBD patients had a significantly higher risk of colonoscopy-associated perforation with older age, female gender and therapeutic procedures as main risk factors for perforation [3].

Our case is remarkable for several reasons: Firstly, the patient in this report had mucosal healing on high definition white

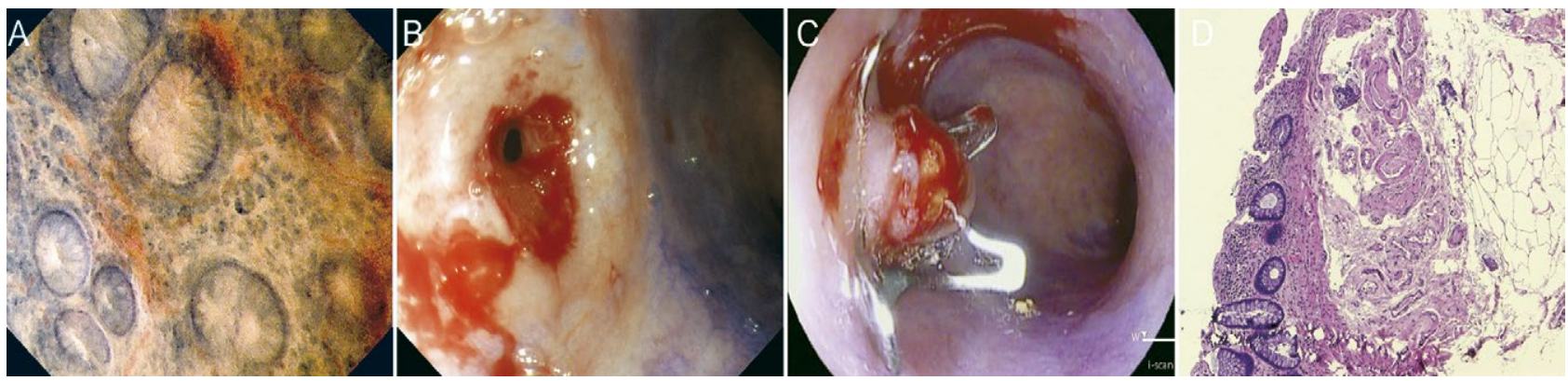

Fig. 1. Prior to biopsy, endocytoscopy showed crypt distortion, crypt distention, accumulation of inflammatory cell infiltrates and hypervascularization in the lamina propria of the sigmoid colon (A). After biopsy with standard sized forceps, a small perforation occurred at the biopsy site (B) and could be successfully closed by over the scope-clipping (C). Similar to endocytoscopy, conventional histopathology showed crypt irregularity and presence of inflammatory cells as well as an almost entire atrophy of the colonic wall (D). 
light endoscopy, and thereby this case extends the common notion that most perforations occur in IBD patients with endoscopic disease activity $[3,4]$. Secondly, our patient had steroid dependent disease and most likely, fibrosis and atrophy of the lamina propria, as confirmed by histopathology, together with steroid use, led to a certain fragility of the colonic wall, leading to perforation after standard forceps biopsy. Further, our case exemplifies that biopsy-related perforations in IBD patients can be successfully managed by OTSC, a technique that has been recently introduced for perforations of the gastrointestinal tract. Reports on the use in IBD patients are rare and hence, OTSC should be cautiously used in patients with more severe inflammation, as the tissue might be harder to grasp with the progression of inflammation. Thirdly, although high definition colonoscopy showed mucosal healing, EC clearly revealed distinct microscopic inflammation. This observation is consistent with data in the literature showing that microscopic inflammation as visualized with EC shows strong correlation with histopathology [5] and that EC can assess histological healing in patients with UC without the need for biopsy specimens [6]. Further, in a functional context, data suggest that endocytoscopic grading of inflammatory activity can act as a predictive indicator for disease relapse [7] and when combined with technologies of artificial intelligence, EC can provide fully automated identification of persistent histologic inflammation [8].

Therefore, as exemplified in our case report, advanced imaging techniques, especially those being able to analyse the mucosa on a cellular level (like EC), present a non-invasive and precise "optical biopsy" compared to physical biopsies for histological assessment of disease activity and can lead to a more precise grading of inflammatory activity during endoscopy.

Entcho Klenske ${ }^{1}$, Arndt Hartmann ${ }^{2}$, Raja Atreya ${ }^{1}$, Markus F. Neurath ${ }^{1}$, Timo Rath ${ }^{1}$

1) Department of Medicine 1, Ludwig Demling Endoscopy Center of Excellence, University Hospital of Erlangen, Erlangen; 2) Institute of Pathology, University Hospital of Erlangen, Erlangen, Germany

Correspondence: Timo Rath, timo.rath@uk-erlangen.de

\section{Conflicts of interest: None.}

Acknowledgements: The authors acknowledge the help of the endoscopy staff in specimen collection.

DOI: $10.15403 /$ jgld-2267

\section{REFERENCES}

1. Eckardt VF, Gaedertz C, Eidner C. Colonic perforation with endoscopic biopsy. Gastrointest Endosc 1997;46:560-562. doi:10.1016/s00165107(97)70017-8

2. Miyatani H, Yoshida Y. Retroperitoneal perforation caused by endoscopic biopsy in a patient with ulcerative colitis and cytomegalovirus infection. Endoscopy 2007;39 Suppl 1:E246. doi:10.1055/s-2007-967010

3. Navaneethan U, Parasa S, Venkatesh PG, Trikudanathan G, Shen B Prevalence and risk factors for colonic perforation during colonoscopy in hospitalized inflammatory bowel disease patients. J Crohns Colitis 2011;5:189-195. doi:10.1016/j.crohns.2010.12.005

4. Makkar R, Bo S. Colonoscopic perforation in inflammatory bowel disease. Gastroenterol Hepatol (N Y) 2013;9:573-583.

5. Bessho R, Kanai T, Hosoe N, et al. Correlation between endocytoscopy and conventional histopathology in microstructural features of ulcerative colitis. J Gastroenterol 2011;46:1197-1202. doi:10.1007/s00535-011-0439-1

6. Nakazato Y, Naganuma M, Sugimoto S, et al. Endocytoscopy can be used to assess histological healing in ulcerative colitis. Endoscopy 2017;49:560-563. doi:10.1055/s-0043-106556

7. Nishiyama S, Oka S, Tanaka S, et al. Clinical usefulness of endocytoscopy in the remission stage of ulcerative colitis: a pilot study. J Gastroenterol 2015;50:1087-1093. doi:10.1007/s00535-015-1059-y

8. Maeda Y, Kudo SE, Mori Y, et al. Fully automated diagnostic system with artificial intelligence using endocytoscopy to identify the presence of histologic inflammation associated with ulcerative colitis (with video). Gastrointest Endosc 2019;89:408-415. doi:10.1016/j.gie.2018.09.024

\section{Over-the-scope clip closure indicated as first- line therapy for iatrogenic rectal perforation}

\section{To the Editor,}

Retroflexion of the colonoscope is considered an essential part due to the diagnostic ability of the retroflexed view over the forward view [1]. However, retroflexion injury such as rectal perforations occurs because of mechanical trauma of the scope maneuver [2, 3]. First-step attempted hemoclips sometimes fail to completely close after perforation therefore requiring surgical repair. However, emergency surgery has not yielded good results for patients in poor general condition. Therefore, less-invasive instruments are needed to be developed in order to compensate the gap between endoscopic treatment and surgery. The over-the-scope clip (OTSC) (Ovesco Endoscopy AG, Tübingen, Germany) has currently emerged as a new alternative for handling refractory gastrointestinal diseases. The OTSC system is a full-thickness clipping device designed for flexible endoscopes, intended for the wound closure of bothersome diseases including non-variceal bleeding, perforation, fistula, and anastomotic dehiscence. It has several advantages of quick and simple usage and also sustained tissuegrasping force compared with the conventional hemoclips. A literature review of 1.517 cases for OTSC use provided a satisfactory clinical success rate of $78.3 \%$ (range 53-100) overall, showing the successful rates for each indication as follows: $85 \%$ for bleeding, $85 \%$ for perforation, $52 \%$ for fistulas, and $66 \%$ for anastomotic dehiscence, respectively [4]. While the OTSC has a limited ability for closing indurated tissues shown in fistula and anastomotic dehiscence, it demonstrates the maximum effect for immediate closure of non-indurated tissues in bleeding and perforation. Especially, OTSC can be a more suitable first-line option for managing iatrogenic perforation in which an approximately $10 \mathrm{~mm}$ of tear occurs due to scope maneuver. Haito-Chavez et al. [5] reported that the OTSC closure was successful in all of 14 cases with iatrogenic colorectal perforation. However, the choice as first 
or second-line therapy remains uncertain. We herein describe a notable case in which OTSC can be a first-line therapeutic option for an iatrogenic rectal perforation.

A 73-year-old male underwent routine colonoscopy. A perforation measuring $12 \mathrm{~mm}$ occurred at the recto-sigmoid junction located above the peritoneal reflection when straightening the scope after a retroversion maneuver. The closure using hemoclips (HX-610-090; Olympus, Tokyo) was unsuccessful, thus the patient was referred to our hospital for OTSC closure. The following procedure was performed after obtaining informed consent. At first, the implanted hemoclips were attempted to be removed using grasping forceps. Unfortunately, several hemoclips deviated into the mesocolon (Fig. 1A). Although this difficult situation needed the longer procedure time (26 minutes), the removal of all hemoclips succeeded under X-ray assistance. After removing all hemoclips, we could obtain the clear view of the perforation site (Fig. 1B). Simple suction was used to aspirate the defect into the cap (Fig. 1C), and the defect was completely closed by one OTSC ( $\mathrm{t}$ type, $11 \mathrm{~mm}$ ) without procedure-associated complications (Fig. 1D). The total procedure time was 30 minutes. Seven days later, an X-ray examination showed no leakage of the perforation site. Eventually, emergency surgery could be avoided, and fifteen days later, this patient was discharged from our hospital.

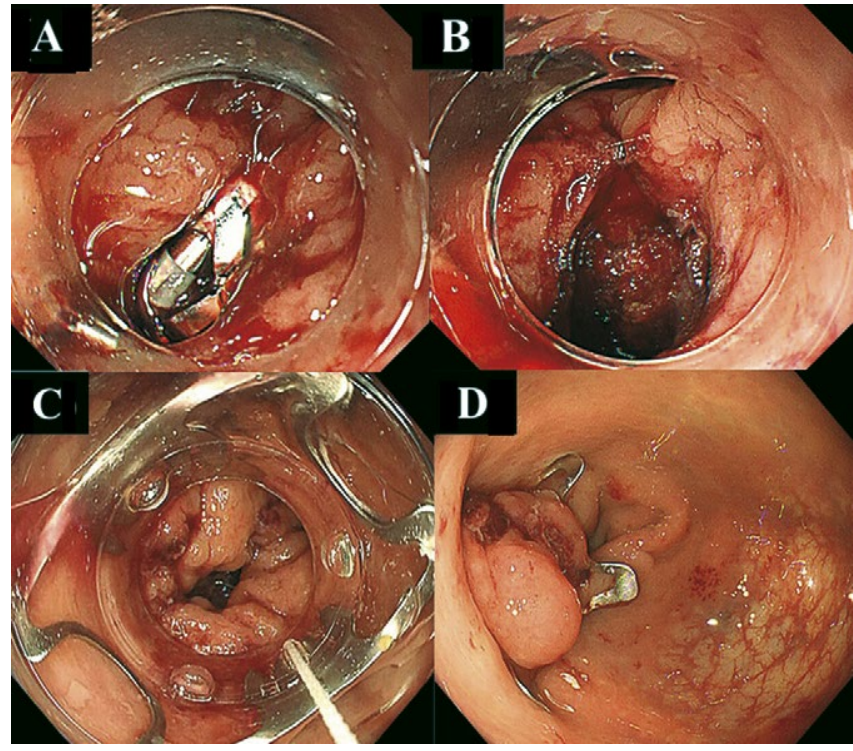

Fig.1. A) Attempted hemoclips deviating into the peritoneal cavity at an iatrogenic perforation (12 $\mathrm{mm}$ in diameter) site due to retroflexion of the colonoscope; B) Clear view of the perforation site after removing all hemoclips; C) Simple suction by one OTSC (t type, $11 \mathrm{~mm}$ ); D) Complete closure by OTSC deployment.

Six months later, the surveillance colonoscopy revealed the spontaneous migration of OTSC, and the healing scar.

This case suggests OTSC might be a reliable first-line therapy for managing an immediate iatrogenic perforation related to a scope maneuver.

Tatsuo Yachida ${ }^{1,2}$, Hideki Kobara ${ }^{1}$, Noriko Nishiyama ${ }^{1}$, Tadayuki Takata $^{1,2}$, Hisashi Masugata ${ }^{2}$, Tsutomu Masaki ${ }^{1}$
1) Department of Gastroenterology and Neurology, Faculty of Medicine, Kagawa University, Kagawa; 2) Department of General Internal Medicine, Faculty of Medicine, Kagawa University, Kagawa, Japan

Correspondence: Tatsuo Yachida, tyachida@med.kagawa-u.ac.jp

Conflicts of interest: None.

DOI: $10.15403 /$ jgld-2483

\section{REFERENCES}

1. Varadarajulu S, Ramsey WH. Utility of retroflexion in lower gastrointestinal endoscopy. J Clin Gastroenterol 2001;32:235-237. doi:10.1097/00004836-200103000-00012

2. Quallick MR, Brown WR. Rectal perforation during colonoscopic retroflexion: a large, prospective experience in an academic center. Gastrointest Endosc 2009;69:960-963. doi:10.1016/j.gie.2008.11.011

3. John AK, Al Kaabi S, Thandassery RB, Dweik N. Retroflexion-induced perforation during colonoscopy after polypectomy: a word of caution. Endoscopy 2015;47 Suppl 1:E186. doi:10.1055/s-0034-1365387

4. Kobara H, Mori H, Nishiyama N, et al. Over-the-scope clip system: A review of 1517 cases over 9 years. J Gastroenterol Hepatol 2018;34:2230. doi:10.1111/jgh.14402

5. Haito-Chavez Y, Law JK, Kratt T, et al. International multicenter experience with an over-the-scope clipping device for endoscopic management of GI defects (with video). Gastrointest. Endosc 2014;80:610-622. doi:10.1016/j.gie.2014.03.049

\section{A case of emphysematous gastritis related to cocaine use treated successfully with conservative management}

\section{To the Editor,}

Emphysematous gastritis (EG) is a rare and potentially fatal gastrointestinal disease characterized by gastric pneumatosis caused by gas-forming bacteria. The most frequently isolated organisms include Escherichia coli, Streptococci species, Enterobacter species, Clostridium perfringens and Pseudomonas aeruginosa [1]. Previously reported risk factors include ingestion of corrosive substances, alcohol use, recent abdominal surgery, pancreatitis and nonsteroidal antiinflammatory drug use [2]. Emphysematous gastritis may present with non-specific symptoms including abdominal pain, nausea, vomiting and hematemesis. Significant morbidity and mortality with an estimated mortality rate of $50-60 \%$ has been reported for EG [3]. While this condition has been previously reported in the literature, this is the first reported case of EG in the setting of recent drug use. We present a case of EG in the setting of cocaine use that resolved with early and appropriate conservative management.

A 33-year-old Caucasian female with a past medical history of illicit drug use (cocaine and heroin) and chronic liver disease secondary to hepatitis $\mathrm{C}$ virus presented with severe epigastric pain, nausea and non-bloody non-bilious 
vomiting in the setting of recent drug use. She was a chronic illicit drug user who has snorted cocaine since she was 16 years old, with recent daily use prior to admission. On physical exam, she was cachectic, ill-appearing, and in mild respiratory distress. Urine drug screen was positive for cocaine. Computed tomography (CT) of the abdomen revealed air in the gastric wall at the fundus, suggestive for EG (Fig. 1). Upper endoscopy was not performed due to concern for perforation. Given the lack of peritoneal signs, conservative management was proposed, using bowel rest, intravenous fluids and Piperacillin/ Tazobactam therapy. She improved clinically after completing a seven-day course of antibiotics; the follow-up CT demonstrated complete resolution of air in the gastric wall.

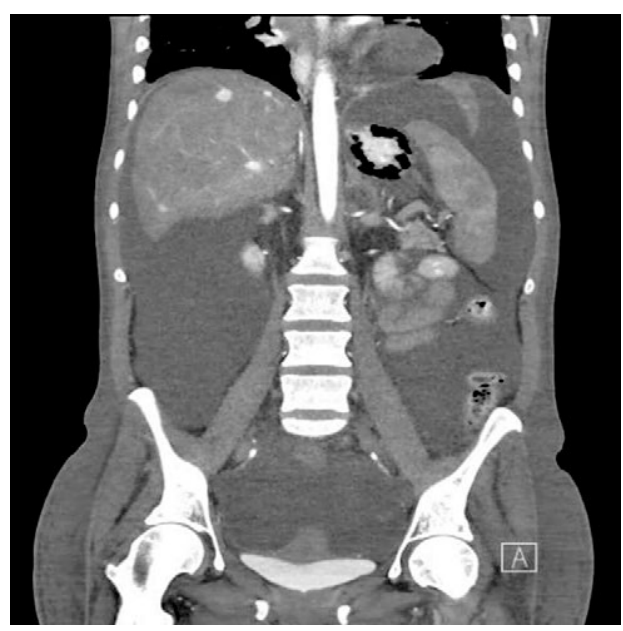

Fig. 1. Air in the gastric wall at the fundus.

In the presented case, cocaine might have disturbed the integrity of the gastric wall, secondary to ischemia, predisposing the patient to infection with gas-forming bacteria. Many factors have been associated with the alteration of this barrier, including ingestion of corrosive substances, alcohol abuse, recent abdominal surgery, and gastric infarction. Cocaine use can lead to various gastrointestinal complications including bowel ischemia, infarction, and perforation. The patient had none of the prior reported predisposing risk factors for EG and therefore cocaine appears to be the most likely contributing factor.

Conservative management of EG includes fluid resuscitation and broad-spectrum antibiotics covering gram-negative and anerobic organisms. Diagnosis is made with an abdominal CT scan due to the high sensitivity and specificity of demonstrating pockets of air throughout the stomach wall [4]. It is important to differentiate EG from gastric emphysema, a noninfectious cause of air in the gastric mucosa. These patients appear nontoxic and imaging reveals thin, linear lucencies in the gastric mucosa [4]. Our diagnosis was made based on the CT imaging and clinical findings of severe gastrointestinal symptoms and sepsis. If patients improve clinically, an endoscopy is not needed for diagnosis and treatment. The role for surgical intervention is not well-established and is reserved for those who fail optimal medial management or develop perforation and necrosis. One study comparing cases of EG before and after 2000 revealed that patients with EG before 2000 were about three times more likely to undergo surgical intervention, and that subjects with EG after 2000 had lower rates of mortality (33.3\% vs $59.4 \%$ ) [5]. This decrease in mortality rate has been attributed to an increasing trend towards more conservative management. Given the significant morbidity and mortality, clinicians should maintain a high suspicion for EG in patients with recent drug use and abdominal imaging suggesting air in the gastric wall.

Michael Makar ${ }^{1}$, Taeyang Park ${ }^{1}$, Evan Orosz ${ }^{2}$, Cindy Law ${ }^{2}$, Anish Vinit Patel ${ }^{2}$

1) Department of Internal Medicine, Rutgers Robert Wood Johnson Medical School, New Brunswick, New Jersey 2) Department of Gastroenterology, Rutgers Robert Wood; Johnson Medical School, New Brunswick, New Jersey, USA

Correspondence: Michael Makar, Michaelmakar5@gmail.com

Conflicts of Interest: None.

DOI: $10.15403 /$ jgld-2501

\section{REFERENCES}

1. Szuchmacher M, Bedford T, Sukharamwala P, Nukala M, Parikh N, Devito P. Is surgical intervention avoidable in cases of emphysematous gastritis? A case presentation and literature review. Int J Surg Case Rep 2013;4:456-459. doi:10.1016/j.ijscr.2012.12.021

2. Shipman PJ, Drury P. Emphysematous gastritis: case report and literature review. Australas Radiol 2001;45:64-66. doi:10.1046/j.14401673.2001.00878.x

3. Takano Y, Yamamura E, Gomi K, et al. Successful conservative treatment of emphysematous gastritis. Intern Med 2015;54:195-198. doi:10.2169/ internalmedicine.54.3337

4. Nemakayala DR, Rai MP, Rayamajhi S, Jafri SM. Role Of Conservative Management In Emphysematous Gastritis. BMJ Case Rep 2018;2018:bcr2017222118. doi:10.1136/bcr-2017-222118

5. Watson A, Bul V, Staudacher J, Carroll R, Yazici C. The predictors of mortality and secular changes in management strategies in emphysematous gastritis. Clin Res Hepatol Gastroenterol 2017;41:e1e7. doi:10.1016/j.clinre.2016.02.011

\section{Prolonged use of propolis can increase liver enzymes}

To the Editor,

Drug induced liver injury can determine a great diagnostic challenge as a result of its long list of potential offending causes which can be either prescribed or as over-the-counter medications, such as medicinal herbs and other remedies. Propolis is not listed as one of the causes. Currently, there is no dose recommendation because the product is considered safe. The propolis features are listed in Table I. Here, we report a case of a young man who presented with increased liver enzymes due to chronic ingestion of large doses of propolis.

A 30-year old male was referred to our gastroenterology unit for persistent abnormal liver enzymes over a period of six months. Three months prior to the current presentation, 


\begin{tabular}{ll} 
Table I. Propolis features & \\
\hline Description & - Resinous mixture produced by honey bees mixing saliva and beeswax with exudate gathered from tree \\
& buds, sap flows, or other botanical sources \\
& - Chemical composition depends on environmental conditions -Contains $>300$ compounds \\
\hline Alleged Properties & - Antibacterial \\
& - Antiviral \\
& - Antifungal \\
& - Anti-inflammatory \\
& - Wound healing due to antibacterial properties [1] \\
& -A topical alcoholic extract was more effective than a steroid cream in reducing mast cells (associated with \\
& inflammation and slowed wound healing) in oral surgery wounds [2] \\
& - Topical application was useful to heal cold sores and protect against subsequent breakouts [3] \\
& - Blocking signaling pathways among cancer cells [4] -Allergic contact dermatitis by a shampoo that \\
& contained propolis [5]
\end{tabular}

he experienced recurrent fever, sore throat and fatigue; blood tests showed elevated liver enzymes together with signs of acute Epstein Barr virus (EBV) infection (IgM positivity). For this reason, EBV related acute hepatitis was suspected. At subsequent exams, liver enzymes were persistently elevated, and the patient continued to present fatigue and a sore throat in absence of abdominal pain, vomiting or altered bowel habit. It should be noted that several ear-nose-throat investigations for his sore throat had been repeatedly unremarkable.

Upon presentation, the patient looked in general good health. He declared episodic moderate alcohol consumption but denied controlled substances abuse and smoke. He was taking escitalopram for episodic panic attacks. His abdomen was soft and not tender, and there was no palpable mass or organomegaly. At abdominal ultrasound the liver and the spleen were normal.

The blood tests showed elevated liver enzymes (AST: 88 U/l, ALT: 184 U/l); bilirubin, gamma glutamil transpeptidase, and alkaline phosphatase were within normal ranges. All other parameters from full blood count and renal function were within normal ranges. Work-up for various infective causes of increased liver enzymes such as viral hepatitis (HAV, HBV, $\mathrm{HCV}, \mathrm{HEV}$ ), HIV and cytomegalovirus were negative. EBV IgG were positive while IgM were negative. Autoimmune profiles came back negative. Alpha-1 antitrypsin was normal. Iron or copper storage diseases and coeliac disease were excluded. Escitalopram was withdrawn in view of its possible etiological role in the pathogenesis of increased liver enzymes. However, liver enzyme elevation persisted at subsequent 2 -week and 6 -week controls. Liver biopsy was proposed but the patient refused.

Upon further questioning, it was found that the patient had been taking a large amount of propolis candies ( $>10$ per day) for several months in the attempt to calm his sensation of sore throat. His propolis candies contained a molecular complex of resins "propolgemma", polysaccharides and lignins (obtained from Popolus nigra L.), titrated in total flavonoids $\geq 15 \%$ and polysaccharides $\geq 4 \%$. Other excipients were vegetable glycerin, apple juice, arabic gum, lemon juice, natural cherry and strawberry flavor. As a last attempt, we asked the patient to quit the consumption his propolis candies. To our utter surprise, at 8-week follow up, liver enzymes were normalized.
At subsequent 3-month follow up the patient's liver tests remained within normal ranges. He was strongly advised against further propolis consumption.

As there is no single test that can directly determine drug induced liver injury, drug history plays a pivotal role in identifying the cause. This should be done by meticulously enquiring the use of both prescribed and over-the counter medications. The lack of liver histology represents the main limitation of our case study. However, it was possible to reach such an unprecedented diagnosis only after ruling out all common and uncommon causes of liver injury.

More data collection is warranted in order to evaluate the hazardous effect of this bee product when taken in large quantities for a long time.

\section{Maria Cristina D'Ercole, Pietro Fusaroli}

Gastroenterology Unit, Department of Medical and Surgical Sciences, Hospital of Imola, University of Bologna, Italy

Correspondence: Maria Cristina D’Ercole; m.dercole@ausl.imola.bo.it

Conflicts of interest: None.

DOI: $10.15403 /$ jgld-2582

\section{REFERENCES}

1. Eskandarinia A, Kefayat A, Gharakhloo M, et al. A propolis enriched polyurethane-hyaluronic acid nanofibrous wound dressing with remarkable antibacterial and wound healing activities. Int J Biol Macromol 2020;149:467-476. doi:10.1016/j.ijbiomac.2020.01.255

2. Barroso PR, Lopes-Rocha R, Pereira EM, et al. Effect of propolis on mast cells in wound healing. Inflammopharmacology 2012;20:289-294. doi:10.1007/s10787-011-0105-5

3. Sforcin JM. Biological Properties and Therapeutic Applications of Propolis. Phytother Res 2016;30:894-905. doi:10.1002/ptr.5605

4. Chan GC, Cheung KW, Sze DM. The immunomodulatory and anticancer properties of propolis. Clin Rev Allergy Immunol 2013;44:262-273. doi:10.1007/s12016-012-8322-2

5. Navarro-Triviño FJ, Ruiz-Villaverde R. Allergic contact dermatitis of head and neck by propolis contained in a shampoo. Contact Dermatitis 2020;82:409-410. doi:10.1111/cod.13491 


\section{COVID-19 in a young liver transplant recipient: caution for drug-drug interactions}

\section{To the Editor,}

The pandemic outbreak of severe acute respiratory syndrome Coronavirus 2 (SARS-CoV-2), and its associated disease (COVID-19), is greatly affecting the management of patients with liver disease. In particular, immunosuppressed patients, such as liver transplant recipients, are a susceptible category at a high risk of infection. Here, we present the case of a liver transplant patient with COVID-19 and the issues dealt with during antiviral treatment.

A 27-year old transplanted male patient was admitted to the hospital with fever on March 26th, 2020. His medical history evidenced: primary sclerosing cholangitis (PSC), ulcerative colitis, liver transplantation in April 2018 and PSC relapse in July 2019, immunosuppressive therapy with tacrolimus and prednisone. The patient was recently hospitalized for an episode of cholangitis and successfully treated with intravenous antibiotics. Five days before the actual presentation, he had another episode of fever and started oral antibiotics. As he developed ageusia, anosmia, and was in contact with positive SARS-CoV-2 relatives, he was tested and as the result was positive for this infection and admitted to the hospital.

At admission, the patient presented low-grade fever, but no symptoms or signs of respiratory distress. The oxygen saturation in room air was excellent, and the chest X-ray was negative. Antiviral treatment with darunavir/ritonavir and hydroxychloroquine was initiated. Given the well-known drugdrug interactions, the tacrolimus dosage was reduced from 4 $\mathrm{mg}$ to $1 \mathrm{mg}$ per day. On the next day, the tacrolimus level was slightly increased (from $8.2 \mathrm{ng} / \mathrm{ml}$ at admission to $9.5 \mathrm{ng} / \mathrm{ml}$ ); therefore, tacrolimus was suspended. Despite this precautious measure, on March the 30th, the tacrolimus level was found as high as $22 \mathrm{ng} / \mathrm{mL}$. The patient did not present any symptoms; only an acute kidney injury (creatinine increase from $0.46 \mathrm{mg} /$ $\mathrm{dL}$ to $0.91 \mathrm{mg} / \mathrm{dL}$ ) was observed at biochemical tests. Although immunosuppressive treatment was suspended for almost a week, tacrolimus levels were persistently high during the administration of antiviral treatment, (i.e. $14.8 \mathrm{ng} / \mathrm{mL}$ on April the $3 \mathrm{rd}$, last day of darunavir/ritonavir) (Fig. 1). The patient remained mostly afebrile and asymptomatic during hospital stay. At discharge on April,

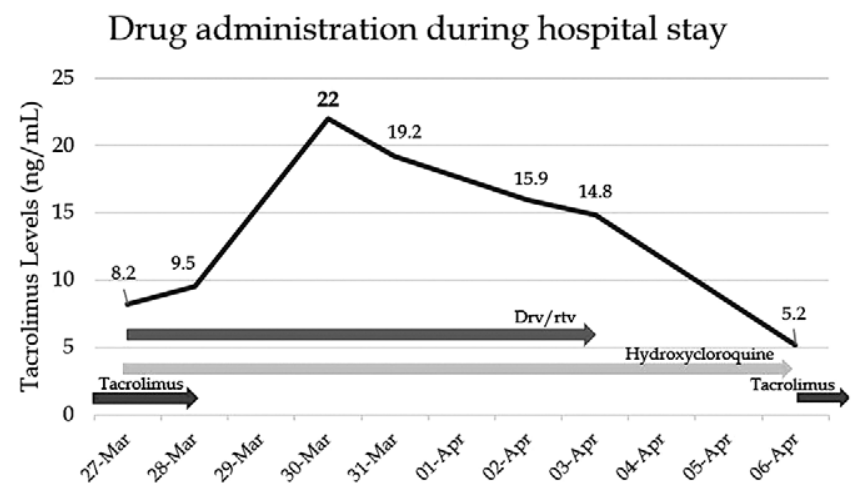

Fig. 1. Levels of tacrolimus during hospital stay in a SARS$\mathrm{CoV}-2$ positive transplanted patient treated with antivirals and hydroxychloroquine. the 6th, tacrolimus levels were within the normal range (5.2 ng/ $\mathrm{mL}$ ), and immunosuppressive treatment was resumed. The test for SARS-CoV-2 infection in the following week resulted negative.

The management of immunosuppressive treatment in patients with COVID-19 presents a major challenge for the clinician, as it requires a "perfect balance" between the risk of graft rejection, chances of virus elimination, attenuation of systemic inflammatory response, risk of bacterial superinfections and drug-drug interactions $[1,2]$. In fact, recently published position papers from transplantation societies have provided controversial recommendations regarding the immunosuppression management and treatment of COVID-19 [2-4]. Similarly, attitudes among clinicians dealing with transplanted COVID-19 patients were highly heterogeneous, as immunosuppressive therapy was either suspended, reduced, or maintained throughout the course disease, with fatal outcomes described for all strategies [5].

In our case, we aimed to maintain the immunosuppressive therapy, upon reducing the tacrolimus dosage given the well-known drug-drug interactions via the cytochrome P450 CYP3A and previous experience in HIV patients. However, its discontinuation became mandatory because of the unpredictable toxic levels of tacrolimus found during hospitalization. We believe that an accurate risk-benefit assessment remains crucial before the initiation of any treatment for COVID-19, considering the peculiar characteristics of solid transplant recipients; alternatives to antiviral treatment should be explored in this category of patients.

Elton Dajti ${ }^{1}$, Francesco Cristini ${ }^{2}$, Giacomo Tamanini ${ }^{1}$, Matteo Cescon ${ }^{1}$, Franco Bazzoli ${ }^{1}$, Mariarosa Tamè ${ }^{1}$

1) Department of Medical and Surgical Sciences (DIMEC), University of Bologna, Bologna; 2) Infectious Diseases Unit, Rimini-Forlì-Cesena Hospitals, Rimini, Italy

Correspondence: Mariarosa Tamè, mariarosa.tame@aosp.bo.it

Conflicts of interest: None.

DOI: $10.15403 /$ jgld-2672

\section{REFERENCES}

1. Shi Y, Wang Y, Shao C, et al. COVID-19 infection: the perspectives on immune responses. Cell Death Differ 2020;27:1451-1454. doi:10.1038/ s41418-020-0530-3

2. Boettler T, Newsome PN, Mondelli MU, et al. Care of patients with liver disease during the COVID-19 pandemic: EASL-ESCMID position paper. JHEP Rep 2020;2:100113. doi:10.1016/j.jhepr.2020.100113

3. Ritschl PV, Nevermann N, Wiering L, et al. Solid Organ Transplantation Programs Facing Lack of Empiric Evidence in the COVID-19 Pandemic: A By-proxy Society Recommendation Consensus Approach. Am J Transplant 2020;20:1826-1836. doi:10.1111/ajt.15933

4. Fix OK, Hameed B, Fontana RJ, et al. Clinical Best Practice Advice for Hepatology and Liver Transplant Providers During the COVID-19 Pandemic: AASLD Expert Panel Consensus Statement. Hepatology 2020 Apr 16. doi:10.1002/hep.31281

5. Huang J, Lin H, Wu Y, et al. COVID-19 in post-transplantation patientsreport of two cases. Am J Transplant 2020;20:1879-1881. doi:10.1111/ ajt.15896 


\section{A case of esophagitis induced by apixaban}

To the Editor,

We herein present a case of esophagitis induced by apixaban. A man in his 60s underwent upper gastrointestinal (GI) endoscopy to investigate the cause of epigastric pain and discomfort which had been getting worse for approximately one year. He had been taking apixaban $5 \mathrm{mg}$ twice a day for more than a year due to non-valvular paroxysmal atrial fibrillation. There were no other medications with gastrointestinal side effects. The upper GI endoscopy revealed longitudinal pinkish mucosal casts adhering to the middle and lower esophagus (Fig. 1A). In addition, reddish mucosal erosions were observed where the pinkish lesions had peeled off (Fig. 1B). He discontinued taking apixaban, switched to another type of oral direct anticoagulant, and continued the oral administration of lansoprazole $15 \mathrm{mg}$ daily. He was advised to drink enough water with medicine and stay seated for at least 30 minutes after taking any medicine to prevent drugs from adhering to the esophageal mucosa. Follow-up upper GI endoscopy after one month revealed a significant improvement in the esophageal mucosa (Fig. 1C). From the above, we considered that apixaban had induced esophagitis in this case.

Dabigatran associated with tartaric acid, as a component of the drug excipient, has been previously reported to induce esophagitis [1,2]. The tartaric acid is not a component of apixaban, but sodium lauryl sulfate in the coating of apixaban is a surfactant that has been reported to be an irritant for human mucous membranes [3], which may therefore play a role in the etiology of esophagitis. Since it was reported that dabigatran-induced esophagitis can harbor squamous cell carcinoma [4], follow-up upper GI endoscopy is important. We should monitor the possibility of esophagitis in apixaban and dabigatran users; educating patients on how to safely take such medications is very important.

\section{Yoshiyasu Kono $^{1}$, Koji Miyahara ${ }^{1}$, Masahiro Nakagawa ${ }^{2}$}

1) Department of Internal Medicine, Hiroshima City Hiroshima Citizens Hospital, Naka-ku, Hiroshima; 2) Department of Endoscopy, Hiroshima City Hiroshima Citizens Hospital, Naka-ku, Hiroshima, Japan

Correspondence: Yoshiyasu Kono, hxnwq178@yahoo.co.jp
Conflicts of interest: None.

DOI: $10.15403 /$ jgld-2685

\section{REFERENCES}

1. Okada M, Okada K. Exfoliative esophagitis and esophageal ulcer induced by dabigatran. Endoscopy 2012;44 Suppl 2 UCTN:E23-E24. doi:10.1055/s-0031-1291503

2. Yoshimitsu M, Sawada T, Kobayashi T, Yamagishi M. Dabigatraninduced Exfoliative Esophagitis. Intern Med 2016;55:1815. doi:10.2169/ internalmedicine.55.6606

3. Marrakchi S, Maibach HI. Sodium lauryl sulfate-induced irritation in the human face: regional and age-related differences. Skin Pharmacol Physiol 2006;19:177-180. doi:10.1159/000093112

4. Zimmer V, Casper M, Lammert F. Extensive dabigatran-induced exfoliative esophagitis harboring squamous cell carcinoma. Endoscopy. 2014; 46 Suppl 1 UCTN: E273-E274. doi:10.1055/s-0034-1365782

\section{Gastrointestinal endoscopy during extracorporeal membrane oxygenation (ECMO) for COVID-19}

\section{To the Editor,}

The COVID-19 pandemic represents so far an unknown challenge for health care systems and health care professionals around the world. Gastroenterologists must provide adequate endoscopic care for patients who urgently need endoscopic diagnosis and treatment. However, because of the virus spread by aerosols and potential transmission via stool, gastrointestinal endoscopy is categorized as a high-risk procedure in terms of virus transmission [1]. First real-world experience from endoscopy units in regions with different COVID-19 incidence has already been published. However, so far there are no dedicated reports presenting data on endoscopies in patients with severe COVID-19 and acute respiratory distress syndrome (ARDS) on extracorporeal membrane oxygenation (ECMO).

Here we report on six emergency endoscopies performed in three patients who were treated at the intensive care unit for severe COVID-19 in April and May 2020. At the time of the five endoscopies, the patients were on veno-venous ECMO (vv-ECMO) because of ARDS. Indications for endoscopies were suspected upper $(n=3)$ and lower $(n=2)$ gastrointestinal

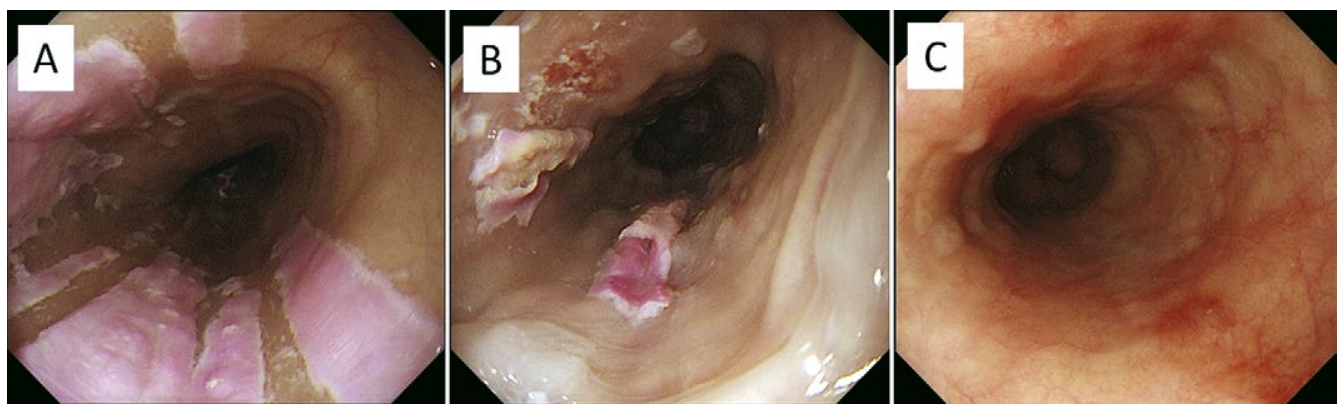

Fig. 1. An endoscopic view of esophagogastroduodenoscopy at the initial point. A) Longitudinal pinkish mucosal casts adhere to the middle and lower esophagus. B) Reddish mucosal erosions were observed where the pinkish lesions had peeled off. C) An endoscopic view of follow-up esophagogastroduodenoscopy on white-light imaging after a month. The pinkish mucosal casts and reddish mucosal erosion had disappeared. 
bleeding as well as paralytic ileus with need for endoscopic decompression $(n=1)$. Whereas signs of recent bleeding were found during three endoscopies (two episodes of diverticular bleed, hemorrhagic gastritis), only subtle changes without signs of active or recent bleeding were observed in two cases. Fig. 1 shows representative images obtained during endoscopies. Prior to endoscopy coagulation was optimized by stopping therapeutic heparinization and supplementing coagulation factors or platelets according to an established protocol for management of bleeding episodes in patients treated with ECMO. Three colonoscopies were incomplete, not reaching the cecum due to insufficient bowel preparation after enema preparation. Endoscopies were performed at our intensive care unit by experienced endoscopists and endoscopy staff within 6 hours of symptom onset for bleeding.

Extracorporeal membrane oxygenation is an established treatment option for patients with ARDS and can also be used for respiratory failure in COVID-19 patients. ECMO is accompanied by a substantial risk of thrombotic complications, which may be aggravated in COVID-19 patients [2]. The need for therapeutic anticoagulation together with an inherent bleeding risk due to multifactorial affection of coagulation by ECMO itself increases the risk for bleeding complications at various sites, including the gastrointestinal tract [3]. Overall, $10-15 \%$ of these patients experience gastrointestinal bleeding episodes during ECMO $[3,4]$. High-dose proton pump inhibitor therapy and optimization of hemostatic derangement following structured protocols are the cornerstones of medical treatment.
Gastrointestinal bleeding is a predictor of worse outcome in patients on ECMO [5]. Thus, in cases of suspected gastrointestinal bleeding, decisions about endoscopy should be made between the intensive care team and endoscopists on a case-by-case basis. Interestingly, endoscopic treatment for gastrointestinal bleeding was not necessary in our series. Notwithstanding, the exclusion of active bleeding and bleeding sites with a high risk of severe re-bleeding may also affect treatment (e.g., continuation of anticoagulation to prevent thromboembolic events).

Here we report that endoscopies can safely be performed in COVID-19 patients with ECMO; hence all necessary endoscopic procedures with potential impact on treatment strategy may be performed. However, there are also specific factors to consider that impede endoscopic procedures in these patients and might impair its quality: loss of image information because of goggles, face shields and isolation screens together with suboptimal lighting conditions in the intensive care unit, atypical positioning of patients as well as endoscopy processors and screens due to medical equipment (ECMO, dialysis machine, ventilator, cannula and tubes), discomfort and limited mobility because of personal protective equipment for the endoscopy team. Given the above-mentioned limitations of endoscopy in the COVID-19 patient with ARDS, the best possible patient preparation (e.g. prokinetics for upper and intensified prograde bowel preparation via nasogastric tube) should be considered.

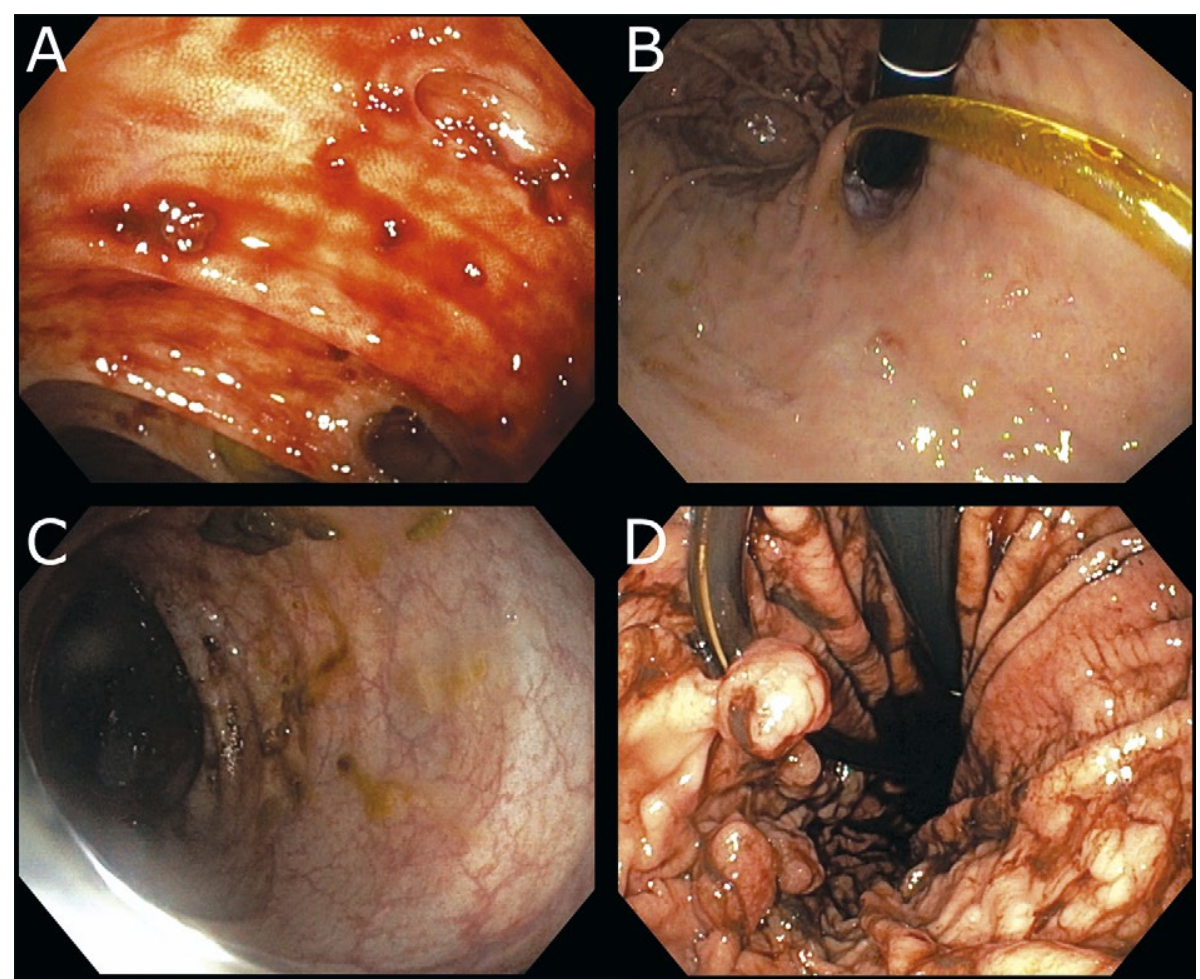

Fig. 1. Endoscopies performed in COVID-19 patients with ARDS. A: Colonoscopy performed in a 60-year-old male recovered from ARDS, on ECMO, day 39 from admission: colonic diverticula with signs of recent bleeding. B: Upper gastrointestinal endoscopy in a 69-year-old male on ECMO, day 22 from admission: slight gastritis and small gastric erosions. C: Colonoscopy on a 55-years male, day 13 from admission: colonic dilation without signs of ischemia. D: Upper gastrointestinal endoscopy in a 55-year-old male, on ECMO, day 26 from admission: hemorrhagic gastritis. Abbreviations: ARDS: acute respiratory distress syndrome; ECMO: extracorporal membrane oxygenation. 
Markus Casper', Philipp M. Lepper², Guy Danziger², Matthias Christian Reichert ${ }^{1}$, Frederik Seiler ${ }^{2}$, Robert Bals ${ }^{2}$, Frank Lammert $^{1}$, Marcin Krawczyk ${ }^{1}$

1) Department of Medicine II: Gastroenterology, Hepatology and Endocrinology, Saarland University Medical Center, Saarland University, Homburg; 2) Department of Medicine V: Pneumology, Allergology and Intensive Care Medicine, ECLS Center Saar, Saarland University Medical Center, Saarland University, Homburg, Germany

Correspondence: Markus Casper, markus.casper@uks.eu

Conflicts of interest: None.

DOI: $10.15403 /$ jgld-2826

\section{REFERENCES}

1. Chen N, Zhou M, Dong X, et al. Epidemiological and clinical characteristics of 99 cases of 2019 novel coronavirus pneumonia in Wuhan, China: a descriptive study. Lancet 2020;395:507-513. doi:10.1016/S0140-6736(20)30211-7

2. Meraviglia P, Schiavini M, Castagna A, et al. Lopinavir/ritonavir treatment in HIV antiretroviral-experienced patients: evaluation of risk factors for liver enzyme elevation. HIV Med 2004;5:334-343 doi:10.1111/j.1468-1293.2004.00232.x

3. Cai Q, Huang D, Yu H, et al. COVID-19: Abnormal liver function tests. J Hepatol 2020;73:566-574. doi:10.1016/j.jhep.2020.04.006

4. Li J, Fan JG. Characteristics and Mechanism of Liver Injury in 2019 Coronavirus Disease. J Clin Transl Hepatol 2020;8:13-17. doi:10.14218/ JCTH.2020.00019

5. Li H, Liu L, Zhang D, et al. SARS-CoV-2 and viral sepsis: observations and hypotheses. Lancet 2020;395:1517-1520. doi:10.1016/S01406736(20)30920-X

\section{SARS-CoV-2 and hepatitis}

\section{To the Editor,}

The novel coronavirus disease (COVID-19) causes a clinical picture with multiple degrees of severity; interstitial pneumonia is the most common complication of the infection, but several other organs can be affected. A liver involvement often manifests itself with mildly and self-resolving hyper-transaminases and hyper-bilirubinemia [1]. Severe hypertransaminasemia occurs rarely. Hereby, we describe a case of acute hepatocellular hepatitis related to COVID-19 and our experience.

A 61-year old Caucasian male was hospitalized for fever, cough and dyspnoea; the throat swab was positive for severe acute respiratory syndrome caused by coronavirus 2 (SARS$\mathrm{CoV}-2)$. The past medical history revealed chronic hepatitis C (grade 2 fibrosis), treated in 2017 and achieving HCV eradication and normalization of liver function tests (LFTs).

At admission laboratory findings showed moderate leucopoenia and thrombocytopenia, mild increase of $\mathrm{C}$ reactive protein and a mild elevation of transaminases $(\mathrm{AST}=67 ; \mathrm{ALT}=102 \mathrm{UI} / \mathrm{L})$. The chest $\mathrm{X}$-ray showed increased interstitial markings and the chest computed tomography revealed diffuse ground glass opacities and bilateral airspace peripheral consolidations. Therapy with lopinavir/ritonavir, chloroquine, methylprednisolone and enoxaparin was started. On the $14^{\text {th }}$ day of hospitalization the patient presented a sudden severe elevation of LFTs (ALT $=1189$. AST $=347$ $\mathrm{UI} / \mathrm{L}$; alkaline phosphatase $=182 \mathrm{U} / \mathrm{L}$; gamma-glutamyl transferase $=108 \mathrm{UI} / \mathrm{L}$; total bilirubin $=17.7 \mathrm{umol} / \mathrm{L}) . \mathrm{No}$ gastrointestinal manifestations such as nausea, vomiting, or diarrhoea were reported. The microbiological investigations for hepatotropic viruses (hepatitis virus A, B, C and E, Epstein Barr virus, cytomegalovirus) were negative and autoimmune and metabolic forms of hepatitis were excluded. Lopinavir/ritonavir, known to be possibly responsible for liver enzyme alteration and liver injury [2], and chloroquine had already been suspended 3 and 4 days before, respectively. A percutaneous liver biopsy was performed. The result of the histologic examination is shown in Fig 1. The detection of SARS-CoV-2 on tissue samples through reverse transcriptionpolymerase chain reaction assay (PCR) resulted positive. In the following days transaminases steadily declined (at discharge: AST $=88 \mathrm{U} / \mathrm{l}, \mathrm{ALT}=330 \mathrm{U} / \mathrm{L}$ ) together with the improvement of respiratory function and radiological picture.

In our experience, LFTs abnormalities are commonly seen in COVID-19 patients. From February to June 2020, 309 patients have been hospitalized for COVID-19 in our department (61\% males, mean age 61$)$. Mild or moderate LFTs alterations have been detected in $4-10 \%$ of the patients, while severe elevations were never found at the time of hospitalization. When we compared LFTs measurements at admission and the highest values obtained during the hospital stay, the proportion of patients with values above the upper normal limit (UNL) increased significantly (Table I). This reflects the data provided by Cai et al [3], who reported that in $11-25 \%$ of patients LFTs rose to more than 3 times the UNL within 2 weeks of hospitalization.

The nature of liver injury in COVID-19 is still debated and needs further evaluations. It could be due to an immune interaction involving intrahepatic cytotoxic T and Kupffer cells. A septic state, drug toxicity and progression of pre-existing liver diseases are other possible causes. Nevertheless, even if evidence is still inconclusive, also a virus-related liver injury cannot be excluded [4].

The histological findings of our patient were non-specific and partly likely to be attributable to drug toxicity. However, the research for SARS-CoV-2 in the liver tissue was positive. Considering the moderate form of disease (the patient did not present signs of shock or other organ failure) that can exclude an indirect liver injury, we suggest that the SARS-CoV-2 itself might be the most likely cause of the transaminases' sudden increase.

It is reasonable to assume that the hepatic alterations encountered in COVID-19 patients are multifactorial. However, the virus itself could also be responsible for a direct cytopathic effect that can eventually lead to a clinical picture of acute hepatitis, a phenomenon called viral sepsis [5]. Molecular biology techniques are useful to determine the extent of the hepatic damage caused by a direct viral action.

Marco Trevenzoli ${ }^{1}$, Aurelio Guarnaccia ${ }^{1}$, Irene Alberici ${ }^{1}$, Matteo Fassan $^{2}$, Eugenia Di Meco ${ }^{1}$, Fabio Farinati ${ }^{2}$, Anna Maria Cattelan 


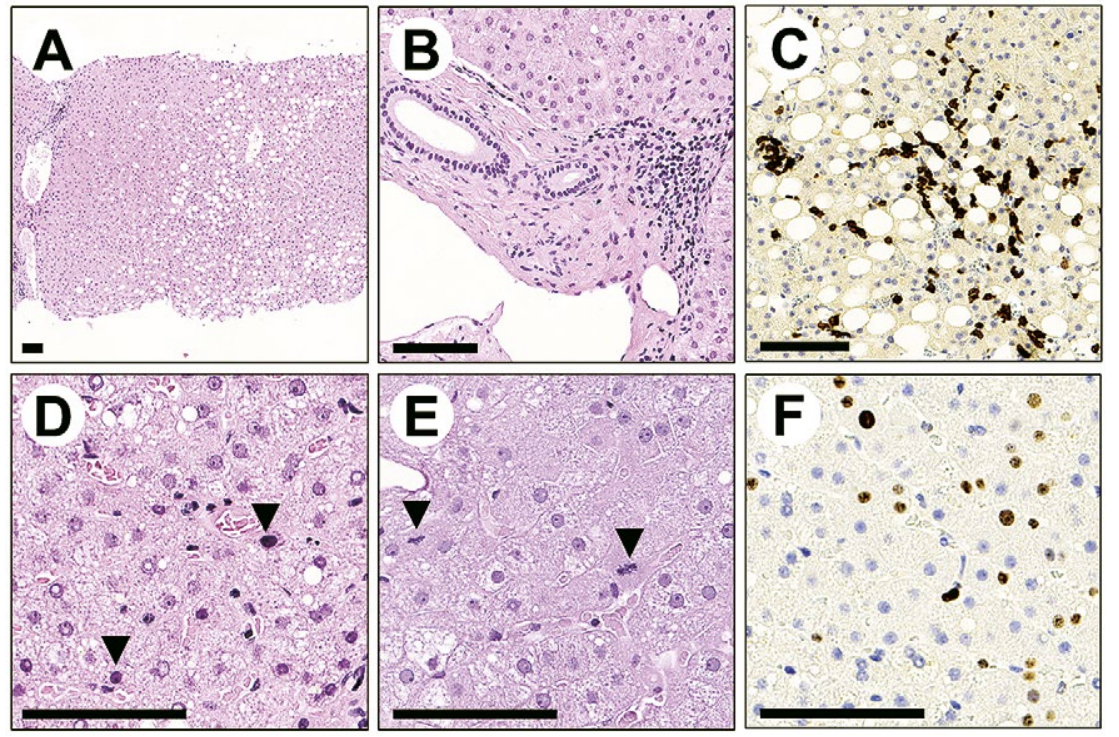

Fig. 1. Histological examination of liver tissue samples $(\mathrm{H} \& \mathrm{E} ;$ scale bar $=100 \mu \mathrm{m}) \mathrm{A}$ : Lobular parenchyma: moderate centrilobular macrovesicular steatosis. B: A representative portal tract: mild lymphocytic inflammation. C: Aggregates of intraparenchyma lymphocytes (CD8 staining). D: Scattered acidophilic bodies (black arrows' heads). E: Occasional mitotic figures (black arrows' heads). F: Ki67-positive regenerating hepatocytes.

Table I. Characteristics of hospitalized patients from February 22 to June 15, 2020 and liver test abnormalities at admission and during hospitalization (highest value obtained)

\begin{tabular}{lccc}
\hline Liver test abnormalities & At admission & During hospitalization & $\mathrm{p}^{*}$ \\
\hline ALT U/L (n.r. $<35)$ & $\mathrm{N}(\%)$ & $\mathrm{N}(\%)$ & \\
Within n.r. & $203(65.9)$ & $120(38.8)$ & $\mathrm{p}<0.01$ \\
$<2$ ULN & $73(23.7)$ & $81(26.2)$ & \\
2 -5 ULN & $29(9.4)$ & $75(24.2)$ & \\
5-10 ULN & $3(1)$ & $25(8.1)$ & \\
$>10$ ULN & 0 & $8(2.6)$ & \\
AST U/L (n.r. $<35)$ & $\mathrm{N}(\%)$ & $\mathrm{N}(\%)$ & \\
Within n.r. & $160(51.8)$ & $109(35.3)$ & $\mathrm{p}<0.01$ \\
$<2$ ULN & $119(38.5)$ & $114(36.9)$ & \\
2 -5 ULN & $24(7.8)$ & $65(21)$ & \\
5-10 ULN & $6(1.9)$ & $19(6.1)$ & \\
$>10$ ULN & 0 & $2(0.6)$ & \\
GGT U/L (n.r. $<65)$ & $\mathrm{N}(\%)$ & $\mathrm{N}(\%)$ & \\
Within n.r. & $244(79.7)$ & $189(61.8)$ & $\mathrm{p}<0.01$ \\
$<2$ ULN & $46(15)$ & $58(18.9)$ & \\
2-5 ULN & $12(3.9)$ & $42(13.7)$ & \\
5-10 ULN & $3(1)$ & $9(2.9)$ & \\
$>10$ ULN & $1(0.3)$ & $8(2.6)$ & \\
TBIL, $\mu$ mol/L (n.r. $<17)$ & $\mathrm{N}(\%)$ & $\mathrm{N}(\%)$ & \\
Within n.r. & $285(92,8)$ & $230(74,9)$ & $\mathrm{p}<0.01$ \\
$<2$ ULN & $18(5,9)$ & $52(16,9)$ & \\
2-5 ULN & $4(1,3)$ & $20(6,5)$ & \\
5-10 ULN & 0 & $5(1,6)$ & \\
\hline ALT & &
\end{tabular}

ALT: alanine aminotransferase; n.r: normal range; ULN: upper limit of normal AST: aspartate transaminase; GGT, gamma glutamyl transpeptidase; TBIL: total bilirubin. ${ }^{*}$ We used the R software (version 3.6.2) to perform $\chi 2$ test to compare differences between the proportions of patients with liver enzymes above the upper limit of normal at admission and during hospitalization. A two-sided $\alpha$ of less than 0.05 was considered statistically significant.

1) Department of Medicine, Infectious Diseases Unit, Azienda Ospedale Università di Padova, Padova; 2) Department of Surgery, Oncology and Gastroenterology, Azienda Ospedale Università di Padova, Padova, Italy.

Correspondence: Anna Maria Cattelan, Annamaria.cattelan@aopd.veneto.it
Conflict of interest: None to declare. DOI: $10.15403 /$ jgld-2747

\section{REFERENCES}

1. Chen N, Zhou M, Dong X, et al. Epidemiological and clinical characteristics of 99 cases of 2019 novel coronavirus pneumonia 
in Wuhan, China: a descriptive study. Lancet 2020;395:507-513. doi:10.1016/S0140-6736(20)30211-7

2. Meraviglia P, Schiavini M, Castagna A, et al. Lopinavir/ritonavir treatment in HIV antiretroviral-experienced patients: evaluation of risk factors for liver enzyme elevation. HIV Med 2004;5:334-343. doi:10.1111/j.1468-1293.2004.00232.x

3. Cai Q, Huang D, Yu H, et al. COVID-19: Abnormal liver function tests. J Hepatol 2020;73:566-574. doi:10.1016/j.jhep.2020.04.006

4. Li J, Fan JG. Characteristics and Mechanism of Liver Injury in 2019 Coronavirus Disease. J Clin Transl Hepatol 2020;8:13-17. doi:10.14218/ JCTH.2020.00019

5. Li H, Liu L, Zhang D, et al. SARS-CoV-2 and viral sepsis: observations and hypotheses. Lancet 2020;395:1517-1520. doi:10.1016/S01406736(20)30920-X

\section{A histopathological observation regarding the possibility of hemophagocytic lymphohistiocytosis in COVID-19 patients}

\section{To the Editor,}

Six months after the onset of the coronavirus disease 2019 (COVID-19) pandemic, there is still much we need to learn about COVID-19's pathogenesis. Some previous studies have suggested that critical cases of COVID-19 with poorer prognoses are characterized by evidence that points to hemophagocytic lymphohistiocytosis (HLH). Secondary HLH is a life-threatening condition that can occur due to the disorder of the immune system's regulation leading to the production of an excessive levels of circulating cytokines, anomalous histiocytic proliferation, and hemophagocytosis throughout the reticuloendothelial system [1-3].

After acquiring the necessary ethical approval, the following study was conducted to examine the histopathological findings in patients who had passed away due to COVID-19.

Hemophagocytosis in the liver biopsy samples of two cases was observed (Fig. 1). The first patient had presented with fatigue, fever, and a sore throat, ultimately deteriorating towards acute respiratory distress syndrome (ARDS), acute kidney injury and a decreased level of consciousness. He was intubated and received respiratory and hemodynamic support in an intensive care unit but passed away after 6 days. In our pathological examination of this case, foci of hemophagocytosis (erythrophagocytosis and lymphophagocytosis) were evident, and immunostaining for CD68 revealed Kupffer cell hyperplasia in the liver tissue. Additional histopathologic findings included mild inflammation of portal tracts, mild macrovesicular steatosis involving about 30 percent of the lobular area, mild microvesicular changes, and foci of pericellular fibrosis without significant portal fibrosis were also identified.

The second patient had presented with myalgia, abdominal pain, nausea, and vomiting and treatment was prompted by applying atazanavir, hydroxychloroquine, piperacillin/ tazobactam, and ceftriaxone. Later on, the patient was admitted to the intensive care unit after demonstrating ARDS-

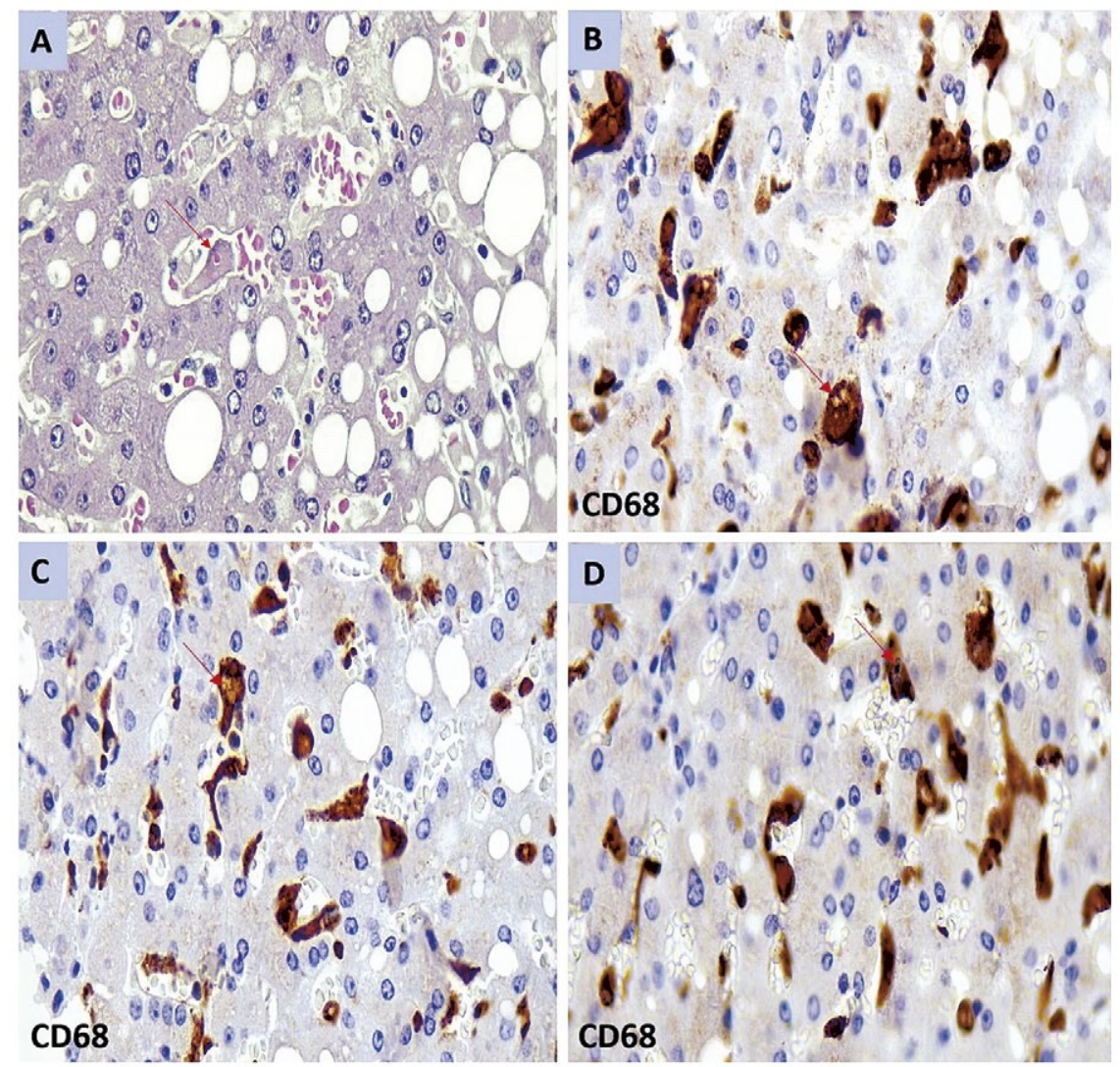

Fig. 1. Pathologic findings in the liver biopsy of COVID-19 cases with hemophagocytosis. A: Erythrophagocytosis by kupffer cells (arrow); B-D: CD68 stained multiple Kupffer cells with hemophagocytosis (arrow). 
related respiratory complications. Despite intubation and hemodynamic support, the patient passed away after 16 days. In our pathological examination, foci of hemophagocytosis were evident, and immunostaining for CD68 highlighted multiple Kupffer cells. Additionally, on portal tracts, mild infiltration of predominantly lymphocytes was observed. Withing lobules, mild focal necrosis (one focus or less per 10x field) was present. Mild to moderate congestion and mild microvesicular changes and macrovesicular steatoses involving about 15 percent of the lobular area were observed. These features can be related to hepatic ischemia after a severe hemodynamic shock. Considering the mild degree of inflammation, other etiologies such as drug reactions and virus-related injuries are less likely.

While conducting a pathological study on 11 randomly selected deceased patients who were confirmed cases of COVID-19, and following a post-mortem core needle liver biopsy procedure, we found the presence of Kupffer cell hyperplasia and hemophagocytosis in the liver of two patients. HLH can be proposed as the cause of such an observation. Among the novel coronavirus-infected patients, cytopenia especially in CD8+ T-cell subsets, increased serum levels of interleukin (IL)-6, IL-10, IL12, and interferon (INF)- $\gamma$, increased serum levels of ferritin, and clinical symptoms such as ARDS, sepsis, and multi-organ failure have been observed [2, 3 ]. While these findings are not unexpected in viral diseases, they might also point to an HLH diagnosis. Viral infections are among the causal triggers of HLH [4]. A sharp increase of IL-10 and INF- $\gamma$ and a moderate increase of IL- 6 are highly indicative of a cytokine profile observed in HLH patients. A course of treatment that has shown promising results in critical cases of coronavirus infection is administering tocilizumab [4]. It is an immunosuppressive drug that inhibits IL-6 and is also adopted in HLH cases in life-threatening instances where it is used to curb interleukin levels [5]. In cases of secondary $\mathrm{HLH}$, if the patient does not suffer from a detectable genetic condition, the treatment process involves administration of dexamethasone, etoposide, and cyclosporine [5].

Alireza Abdollahi ${ }^{1}$, Mohammad Taghi Beigmohammadi ${ }^{2}$, Masoomeh Safaei ${ }^{1}$, Vahid Mehrtash ${ }^{1}$, Bita Jafarzadeh ${ }^{1}$

1) Department of pathology, Imam Hospital Complex, School of Medicine, Tehran University of Medical Sciences, Tehran; 2) Department of Anesthesiology and Intensive Care, Imam Khomeini Hospital Complex, Tehran University of Medical Sciences, Tehran, Iran

Correspondence: Mohammad Taghi Beigmohammadi, mbage46@gmail.com

Conflicts of interest: None.

DOI: $10.15403 /$ jgld-2934

\section{REFERENCES}

1. Chen N, Zhou M, Dong X, et al. Epidemiological and clinical characteristics of 99 cases of 2019 novel coronavirus pneumonia in Wuhan, China: a descriptive study. Lancet 2020;395:507-513. doi:10.1016/S0140-6736(20)30211-7

2. Meraviglia P, Schiavini M, Castagna A, et al. Lopinavir/ritonavir treatment in HIV antiretroviral-experienced patients: evaluation of risk factors for liver enzyme elevation. HIV Med 2004;5:334-343. doi:10.1111/j.1468-1293.2004.00232.x

3. Cai Q, Huang D, Yu H, et al. COVID-19: Abnormal liver function tests. J Hepatol 2020;73:566-574. doi:10.1016/j.jhep.2020.04.006

4. Li J, Fan JG. Characteristics and Mechanism of Liver Injury in 2019 Coronavirus Disease. J Clin Transl Hepatol 2020;8:13-17. doi:10.14218/ JCTH.2020.00019

5. Li H, Liu L, Zhang D, et al. SARS-CoV-2 and viral sepsis: observations and hypotheses. Lancet 2020;395:1517-1520. doi:10.1016/S01406736(20)30920-X 